\title{
Clinicoetiological Study of Epilepsy in Children Presenting To Speciality Epilepsy Clinic at Tertiary Medical Hospital
}

\author{
Zankhana R. Parekh ${ }^{1}$, Umesh Joshi' ${ }^{2}$, Nitish Vora ${ }^{3}$, Aasheeta Shah ${ }^{4}$ \\ ${ }^{1}$ Assistant professor, Department of Paediatrics, NHL Municipal Medical College, ${ }^{2}$ Resident, Department of Paediatrics, NHL Municipal Medical College, ${ }^{3} \mathrm{Paediatric}$ \\ Neurologist, NHL Municipal Medical College, ${ }^{4}$ Professor and HOD, Department of Paediatrics, NHL Municipal Medical College.
}

\section{Abstract}

Background: Objectives: To identify the clinical \& etiological profile of children and the characteristics of seizures in them along with therapeutic response. Subjects and Methods: All patients who attended the Epilepsy Clinic \& fulfilled the selection criteria were enrolled in study. This is a descriptive study of 18 months \& involved analysis of records of the patients who came to specialty OPD. Three groups were formed accordingly - focal, generalized \& unknown onset with further etiological sub-divisions -Genetic, Structural/Metabolic, Immune, Infectious \& Unknown. Results: In all, 417 patients were studied. The distribution as per clinical presentation was- group I (generalized) $215(58.5 \%$ ) - group II (focal) 154(36.9\%), group III (unknown) 48(4.6\%). The main etiologies were perinatal asphyxia (28.3\%) NHBI (11.4\%) in (structural-metabolic) sub group. In Genetic \& Infectious, Channelopathies (10.5\%) \& Post Meningitis Sequelae (4.7\%)were main etiology. $56.3 \%$ of the patient in group II were on more than 3 AEDs. $14.3 \%$ in group I were weaned of AEDs. $61.4 \%$ patients in group II were having neurodevelopmental Sequelae. EEG revealed abnormal activity in 30(6.2\%) in group I \& 31(19.3\%) in group II. Maximum patient with refractory epilepsy were seen in group III. Conclusion: To have a good management of epilepsy we need to have multi-dimensional classification of epilepsy based on both clinical \& etiological spectrum. Perinatal Asphyxia \& NHBI are one of the most common yet avertible etiologies.

Keywords: Epilepsy, perinatal asphyxia, classification, anti-epileptic drugs, EEG.

Corresponding Author: Dr. Zankhana Parekh, 1, Amar Siddhi Apartment, Opp Pankaj Jain Derasar, Pankaj Society, Bhattha, Paldi, Ahmedabad-380007.

Received: April 2019

Accepted: April 2019

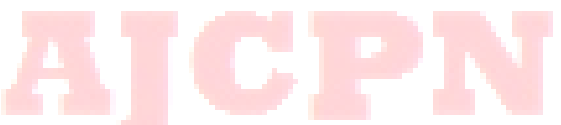

\section{Introduction}

Epilepsy is one of the most urgent problems in pediatric neurology. Asserting the causes and types of seizure is important for diagnostic purposes and for evaluating therapy. In 2010 the ILAE defined focal as "originating within networks limited to one hemisphere. They may be discretely localized or more widely distributed. Focal seizures may originate in subcortical structures." Generalized from onset seizures were defined as "originating at some point within, and rapidly engaging, bilaterally distributed networks." Classifying a seizure as generalized does not rule out a focal onset obscured by limitations of our current clinical methods, but this is more an issue of correct diagnosis than of classification. A working distinction between focal and generalized onset is a practical one, and may change with advances in ability to characterize the onset of seizures. Clinicians have long been aware that so-called generalized seizures, for example, generalized absence with EEG generalized spike-waves, do not manifest equally in all parts of the brain. The ILAE emphasized the concept of bilateral, rather than generalized involvement of some seizures, since seizures can be bilateral without involving every brain network. The term "focal to bilateral tonic-clonic" was substituted for "secondarily generalized." The term "generalized" was maintained for seizures generalized from onset. Clinicians commonly hear about tonic-clonic seizures for which the onset was unobserved. Perhaps, the patient was asleep, alonelor observers were too distracted by the manifestations of the seizure to notice presence of focal features. There should be an opportunity to provisionally classify this seizure even in the absence of knowledge about its origin. The ILAE therefore allowed classification of any seizure type with the modifier "unknown onset." It may be impossible to classify a seizure at all, either because of incomplete information or because of the unusual nature of the seizure. ${ }^{[1]}$

\section{Subjects and Methods}

\section{Study type}

All patients who attended the Epilepsy Clinic \& fulfilled the selection criteria were enrolled in study. This is a descriptive study of 18 months \& involved analysis of records of the 
patients who came to specialty OPD. Three groups were formed accordingly - focal, generalized \& unknown onset with further etiological sub-divisions -Genetic, Structural/Metabolic, Immune, Infectious \& Unknown.

\section{Enrollment Criterion}

- Age less than 14 years at time of presentation to OPD.

- All patients who had seizures in last 1 year time period.

- All patient who are on verge of developing epilepsy.

- All patients presented to our epilepsy clinic based on clinical data combined with EEG and Neuroimaging and other auxiliary diagnosed as symptomatic Epilepsy.

- Febrile seizures were not included

\section{Strength Of Study}

$258(62 \%)$ were male and $159(38 \%)$ were female subjects in our study.

We had further grouped our subjects into age groups in four age-groups.

o 0 - 12 months o 1 year -5 years o 5 years -10 years o $>10$ years

Only in last age group there was female pre-dominance.

\section{Results}

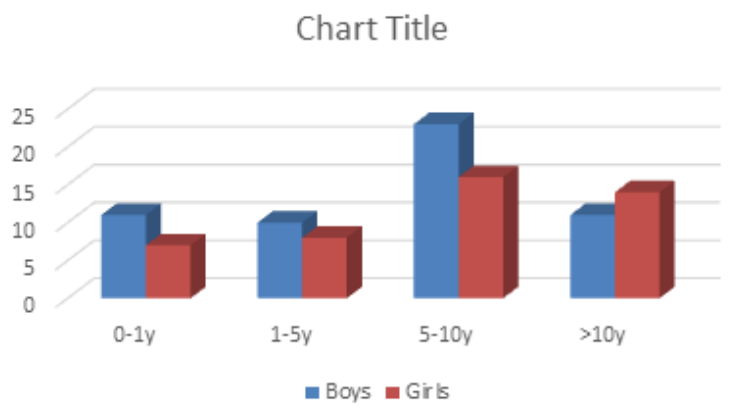

\section{Distribution of study:}

o The distribution according to clinical presentation was as follow o group I (generalized) $215(58.5 \%)$ o group II (focal) 154 (36.9\%) o group III 48 (4.6\%) (unknown).

In sub group Genetic, Channelopathies (10.5\%) and in sub group Infectious, Neuro-cysticercosis and Post Meningitis Sequelae $(4.7 \%)$ were significant etiologies. In structural and metabolic subgroups perinatal asphyxia (hypoxic ischemic encephalopathy) and Neonatal hypoglycemic brain injury (NHBI) were the major etiologies. In Immune subgroup, few cases of auto-immune encephalitis were also there. MTLS was major etiology in unknown/syndromic constellations. MTLS (Mesial Temporal Lobe Sclerosis) was most common in unknown origin group. Neurological examination is usually normal with the exception of memory deficits. Interictal electroencepha-logram (EEG) findings in patients with MTLE typically include unilateral or bilaterally-independent mesial tem-poral spikes, best seen with basal (sphenoidal, inferior tem-poral) derivations. Ictal EEG recordings usually reveal ictal onset consisting of rhythmic 5 to $7 \mathrm{~Hz}$ activity in one mid-inferomesial temporal region, but there may be variations in this pattern. High-resolution MRI often demonstrates unilateral or bilateral hippocampal atrophy associated with hypertintense $\mathrm{T} 2$ signal in one or both hippocampi sometimes extending to amygdala or other medial tempo-ral structures. These findings are highly specific for MTS. Post Tuberculous meningo-encephalitis, development of Epilepsy was also present in our study with $4.7 \%$ (21) patients having sequelae in form of seizure disorder.
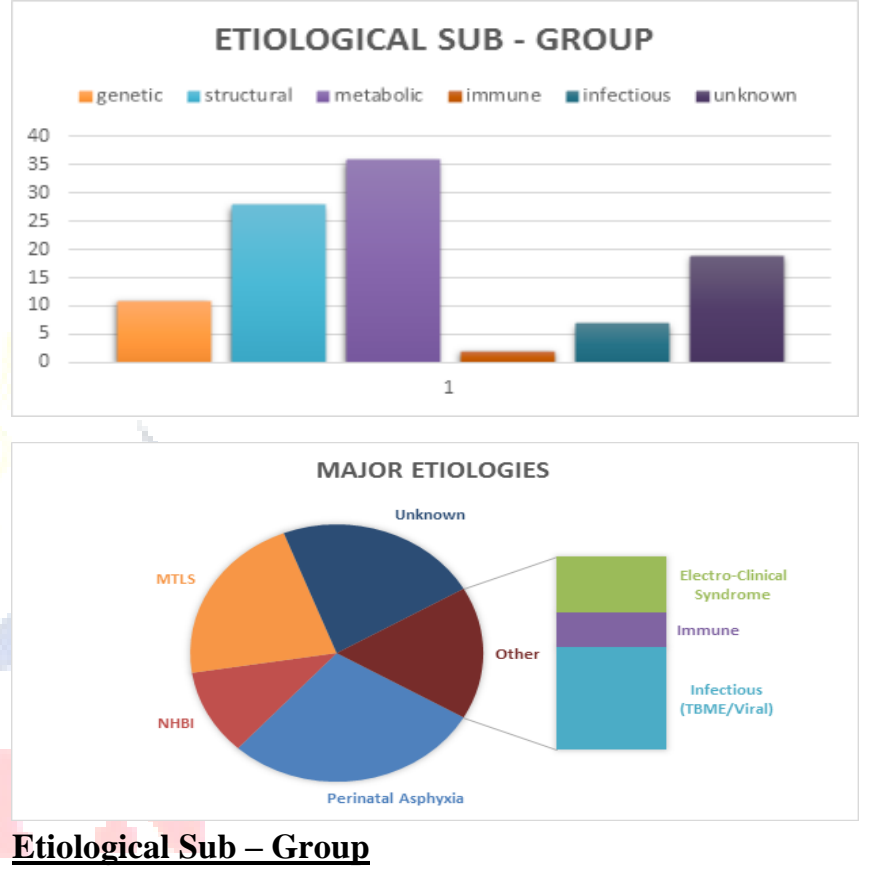

Division according to etiologies

\section{Major Etiologies}

Our study included failure to achieve seizure control with two or more AEDs with adequate dosage and posology. In group I (Generalised), cases of refractory epilepsy were least comparing to other two groups. Sodium Valproate and Carbamazepine being the 1st line therapy, Phenytoin as 2nd line and Leviteracetam/Topiramate/Zonisamide being 3rd line in our facility. In Group I, most of the patients were managed on only Valproate.

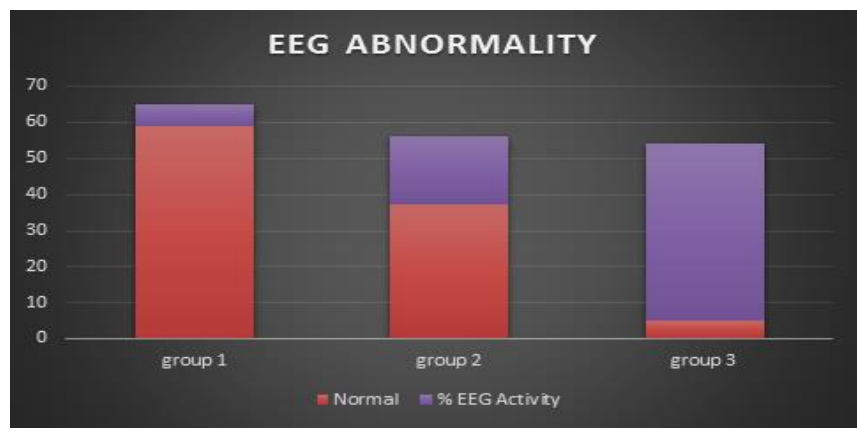




\section{Neurodevelopment Sequelae}

- Normal a Abnormal

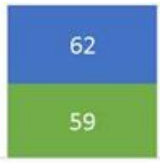

GROUP 1
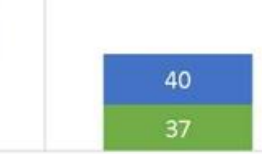

GROUP 2

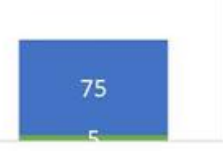

GROUP 3
Neurodevelopment is a term referring to the brain's development of neurological pathways that influence performance or functioning (e.g., intellectual functioning, reading ability, social skills, memory, attention or focus skills).

Neurodevelopment sequelae assessment included gross motor development, behavioral difficulties, speech and personal social domain of development. In Group 3, there was maximum percentage of subjects with such sequelae. In Etiological Sub-group, Metabolic sub group had maximum subjects with such sequelae resulting from perinatal insult mostly.

\section{Response to Therapy}

Patient were weaned off AEDs earlier and successfully in Group 1 (Generalized) in our facility. For Therapeutic response in this, compliance was main key. Most of the patients were in either Valproate alone or Valproate with Leviteracetam along with Clobazam. In group 2, patient were mostly on carbamazepine and valproate combination therapy. There was very little response to the treatment in group 3 (unknown).

\section{Therapeutic Response}

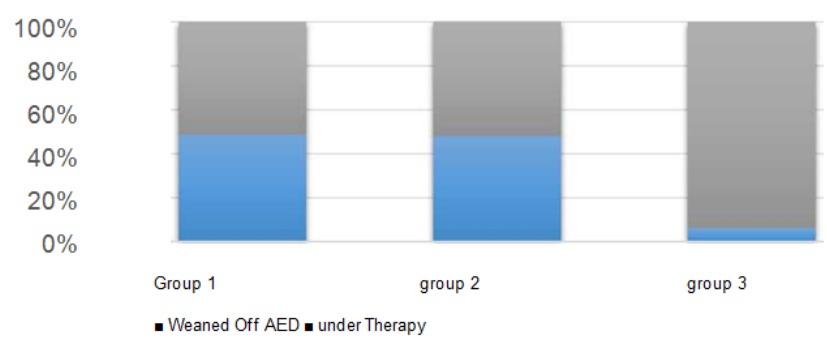

Sodium Valproate is most commonly used AED along with Carbamazepine. Addition of Leviteracetam as 2nd line AED had resulted in better control of seizures in our facility.

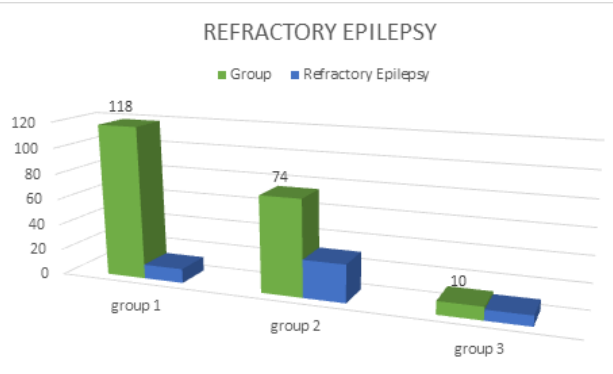

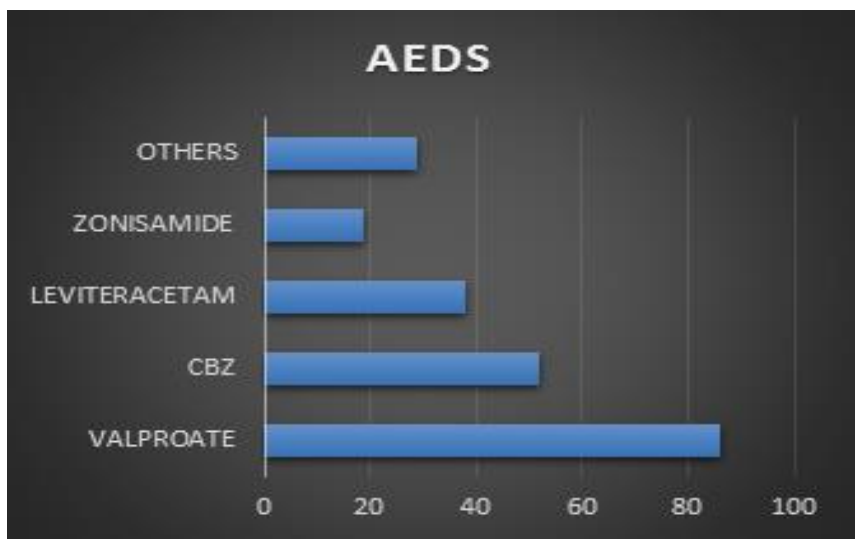

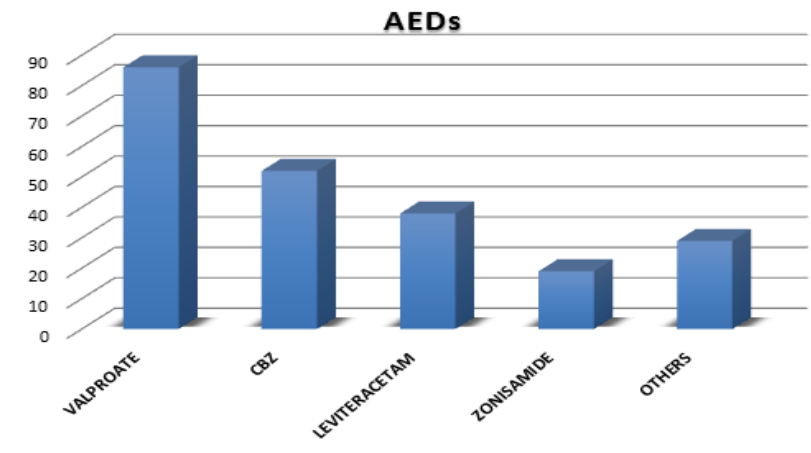

\section{Discussion}

\section{Neonatal Hypoglycemic Brain Injury}

Neonatal hypoglycemia (?46 mg/dL) occurs in $5 \%$ to $15 \%$ of Normal term neonates and can cause visual impairment, epilepsy, and cognitive deficits. Numerous animal and human studies have suggested that neonatal hypoglycemia in the context of HIE may be more detrimental than either condition alone. Neuroimaging studies in neonatal hypoglycemia have shown a correlation between hypoglycemia, parieto-occipital injury, and involvement of the underlying white matter tracts, corpus callosum, and thalamus.

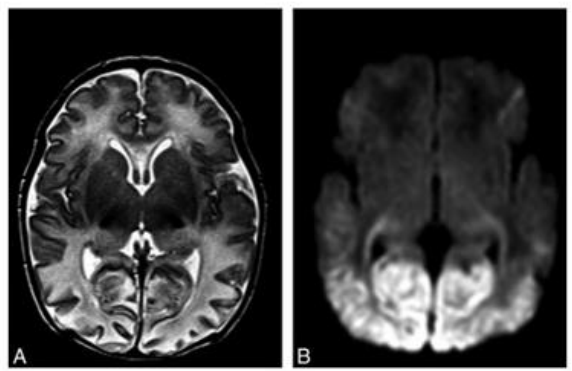

FIG 1. Imaging features of neonatal hypoglycemia superimposed onto HiE. A T2-weighted MR imaging best shows patchy involvement of the anterior medial and pulvinar nuclei of the thalamil Posterior edema and thimning of the cerebral cortex can also be noted 8 . DWI best shows
bilateral restricted diffusion involving the optic radiations and bilateral symmetric edema involving both the parieto-occipital white and gray
matter.

Abnormal imaging patterns can be seen: watershed, basal ganglia, total, and focal-multifocal injury. Prolonged partial asphyxia results in a pattern of injury that primarily involves the watershed zones between the major intravascular 
boundary zones, whereas acute profound asphyxia results in the basal ganglia-predominant pattern of brain injury that involves the basal ganglia, thalami, brain stem, sensorimotor cortex, and corticospinal tracts. Severe watershed and basal ganglia-predominant patterns of injury can manifest as total brain injury, occurring when both the cerebral cortex and deep gray nuclei are hypoperfused. Multifocal pre-dominant pattern of injury describes injury seen in the white matter or cortex. ${ }^{[3,4]}$

\section{Hypoxic ischemic encephalopathy}

A hypoxic-ischemic insult sets in train a cascade of intraneuronal events that may result in cell death. In human neonates, there is often dissociation between clinically evident seizures and seizures recognized on EEG monitoring, so that the frequency of seizure activity after a hypoxic-ischemic insult may well be under-estimated by clinical observation. Therefore, asphyxiated infants are very likely to have a significant seizure burden that may be underestimated by clinicians. Seizures occur in many infants who have sustained a significant hypoxic-ischemic insult indeed, seizure is a feature of moderate and severe HIE. In general, the more severe or prolonged the hypoxia-ischemia, the more seizure activity the infant will have. There has been considerable debate as to whether seizure activity after a hypoxic-ischemic event confers an additional risk factor on the infant in terms of adverse neurodevelopmental outcome.

\section{Outcome on Follow up}

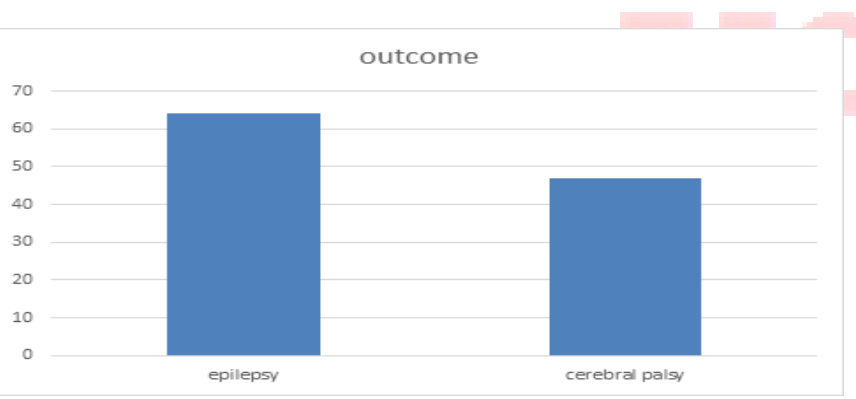

The prognosis for children with HIE depends on the severity and duration of the neurologic abnormality. Major neurodevelopmental problems occur only after moderate and severe HIE, and death is a significant risk in severe HIE.
Emerging data strongly support the observations that a significant number of children with perinatal hypoxicischemic insult previously considered to be without major problems do have significant perceptual-motor difficulties or a reduction in cognitive abilities. ${ }^{[5-8]}$

\section{Conclusion}

To have a good management of epilepsy we need to have multi-dimensional classification of epilepsy based on both clinical \& etiological spectrum. Perinatal Asphyxia \& NHBI both were one of the most common yet avertible etiologies.

Early Breastfeeding of newborn soon after delivery and monitoring of vitals in early neonatal period can alleviate NHBI.

Hypoxic Ischemic Encephalopathy was one of the major cause for epilepsy in our facility. Prognosis depends on multiple factors including early intervention with physiotherapy.

\section{References}

1. Proposal for revised clinical and electroencephalographic classification of epileptic seizures. From the Commission on Classification and Terminology of the International League Against Epilepsy. Epilepsia 1981;22:489-501

2. Harvey AS, Berkovic SF, Wrennall JA, et al. Temporal lobe epilepsy in childhood: clinical, EEG, and neuroimaging findings and syndrome classification in a cohort with new-onset seizures. Neurology. 1997; 49(4):960-968

3. Andre M, Lamblin MD, d'Allest AM, et al. Electroencephalography in premature and full-term infants: developmental features and glossary. Neurophysiol Clin. 2010;40(2):59-124.

4. Barkovich AJ. Injury in the term infant. In: Pediatric Neuroimaging. Philadelphia: Lippincott Williams \& Wilkins; 2005:224-40

5. RolandEH,PoskittK,RodriguezE,etal.Perinatalhypoxic-ischemic thalamic injury: clinical features and neuroimaging. Ann Neurol 1998;44:161-66

6. Richard J. Martin, Fanaroff and Martin's Neonatal-Perinatal Medicine,10th Edition

7. Low JA, et al. Newborn complications after intrapartum asphyxia with metabolic acidosis in the term fetus.Am J Obstet Gynecol. 1994;170:1081-1087

8. Lynch BJ, et al. Natural history and outcome of neonatal hypocalcemic and hypomagnesemic seizures. Pediatr Neurol. $1994 ; 11$

9. Mizrahi EM. Acute and chronic effects of seizures in the developing brain: lessons from clinical experience. Epilepsia. 1999;40(supp11):S42-S50, discussion S64-6.

Copyright: (C) the author(s), 2019. It is an open-access article distributed under the terms of the Creative Commons Attribution License (CC BY 4.0), which permits authors to retain ownership of the copyright for their content, and allow anyone to download, reuse, reprint, modify, distribute and/or copy the content as long as the original authors and source are cited.

How to cite this article: Parekh ZR, Joshi U, Vora N, Shah A. A Study on Outcome of Early Caffeine Administration in Very Preterm Neonates. Asian J. Clin. Pediatr. Neonatol.2019;7(2):9-12.

DOI: dx.doi.org/10.21276/ajcpn.2019.7.2.3

Source of Support: Nil, Conflict of Interest: None declared. 